\title{
Fair intergenerational sharing of a natural resource
}

\author{
Hippolyte d'Albis and Stefan Ambec*
}

September 17, 2009

\begin{abstract}
In this article, overlapping generations are extracting a natural resource over an infinite future. We examine the fair allocation of resource and compensations among generations. Fairness is defined by core lower bounds and aspiration upper bounds. The core lower bounds require that every coalition of generations obtains at least what it could achieve by itself. The aspiration upper bounds require that no coalition of generations enjoys a higher welfare than it would achieve if nobody else extracted the resource. We show that, upon existence, the allocation that satisfies the two fairness criteria is unique and assigns to each generation its marginal contribution to the preceding generation. Finally, we describe the dynamics of such an allocation.
\end{abstract}

\section{Introduction}

Sustainable development is defined by the Brundtland Report as "development that meets the needs of the present without compromising the ability of future generations to meet their own needs". In an economy with natural resources, this definition of sustainable development may require that present generations abstain from extracting any resources. Indeed, as long

*Toulouse School of Economics (INRA-LERNA). We thank two anonymous referees, Franck Portier and participants at seminars held at the Paris, Strasbourg and Aix-Marseille Universities and at the Toulouse Environmental and Resource Economics Conference 2009 for useful comments. This research received financial support from the French National Research Agency (ANR) through the project on "Fair Environmental Policies" and from the chair of "Sustainable Finance and Responsible Investments". 
as a resource is scarce in the precise sense that every generation cannot meet its own resource needs, meeting the needs of present generations would compromise the ability of future generations to meet their own needs. Therefore, natural resource scarcity implies that sustainable development as defined above is impossible.

One way to reconcile the above definition of sustainable development with scarce natural resource is to consider the welfare equivalent of resource needs. Indeed, meeting future generation's needs requires that the present generations reduce their extraction and, therefore, consume less than their needs, which in turn reduces their welfare. Yet they might enjoy as much welfare as if they were to consume the amount necessary to meet their needs if future generations transfer part of their welfare derived from resource extraction. The welfare of present generations who do not extract too much of a resource is then preserved through compensations from future generations. However, the compensation made by future generations to present ones should not be too high, since otherwise, it would compromise their own welfare.

We examine fair paths of extraction and compensations in a natural resource economy by considering two fairness criteria. The first criterion is the so-called core lower bound. It requires that the welfare of any generation or group of generations not be lower than what it could achieve by itself. The theoretical background of this criterion is a fictitious negotiation in which all generations are able to meet, agree on an extraction path and carry out compensations among themselves. We impose the condition that any generation or coalition of generations should obtain at least what it would get as a result of this negotiation.

The second fairness criterion hinges on a solidarity principle and is named the aspiration upper bound. It requires that no generation or group of generations enjoys a welfare higher than its aspiration welfare, i.e. the welfare it would achieve if no other generation were to extract the resource. The scarcity of the resource is here important since, by definition, not all generations can achieve their aspiration welfare. The aspiration upper bound thus features some intergenerational solidarity.

We show that, upon existence, a unique extraction path and vector of compensations satisfy the two fairness criteria. Each generation is assigned a welfare that is equal to its marginal contribution to the preceding generation. We also describe the dynamics of the 
fair extraction path and the compensations. Notably, compensations are shown to increase over time for at least the first generations, which brings into question their feasibility: some generations might not be able to produce enough goods from the resource stock to pay previous generations back. As a consequence, fair allocation might not exist. We provide examples where it does exist and others where it does not. Finally, we show that if there is no technical progress on resource productivity, the welfare of generations decreases over time.

Our paper combines two streams of literature that deal with the management of natural resources in a normative way. On the one hand, axiomatic theory of justice has recently been applied by Bossert et al. (2007), Roemer and Suzumura (2007) and Asheim (2007) in order to compare welfare among generations. On the other hand, dynamic programming methods have been used to solve the social planner's problem, featuring a representative infinitely-lived individual maximizing the sum of a discounted flow of utilities. Pioneer works have been proposed by Dasgupta and Heal (1974) and Solow (1974) for exhaustible resources and these have been extended in many directions. Among them, most notably, is the use of the vintage structure of the population by Marini and Scaramozzino (1995). By combining these two approaches, our fairness axioms thus depend not only on preferences but also on technological constraints and on the resource dynamics. From these axioms, we are then in a position to analyze the fairness properties of extraction paths and intergenerational sharing of welfare.

It should be noted that, in the literature, most of the axioms defining intergenerational fairness treat generations symmetrically. They consider generations behind a "veil of ignorance" with regard to their position on the time scale, which implies that earlier generations should not be favored over later generations and vice versa. Here, in contrast, the two fairness principles treat generations asymmetrically and acknowledge the priority access of the earlier generations to the natural resource. We believe that the sequence of extraction and the dynamics of the resource are two important features of the problem. They characterize the rights and duties of generations in the intergenerational sharing of a natural resource. Concerning the rights, the laissez-faire extraction outcome defines legitimate rights on the resource which, when translated into welfare, lead to the core lower bounds. These rights are de facto resource endowments in an intergenerational exchange economy. By agreeing to reduce 
their resource extraction when young in exchange for a compensation when old, a generation trades part of its resource endowment against consumption. The core lower bounds restrict the allocation of a resource and consumption within the core of this particular exchange economy. In the tradition of the general equilibrium theory, being in the core can be viewed as an attractive fairness principle: a coalition of generations would object to an allocation that is not in the core by arguing that it can achieve a higher welfare by sharing its own endowment among its members. The duties of present generations toward future ones is defined by the solidarity principle. By upper bounding welfare, earlier generations are forbidden from taking advantage of their priority on the resource by getting too much welfare from the trading with future generations. ${ }^{1}$ In a nutshell, the core lower bounds favor earlier generations while the aspiration upper bounds protect later ones.

The paper proceeds as follows: Section 2 introduces the model while Sections 3 and 4 define the fairness principles. In Section 5, we characterize the fair allocation of resources and compensations. We describe its dynamics and discuss its existence in Section 6. Conclusions are given in Section 7.

\section{Model}

A natural resource is exploited by successive overlapping generations indexed by their birth date $t \in \mathbb{N}^{+}$. Let $k_{0}$ be the initial stock of resource and $\rho$ its regeneration rate with $\rho \geq 1$ (the case $\rho=1$ corresponds to an exhaustible resource). Let $x_{t}$ denote the amount of the resource extracted at date $t$. The dynamics of the resource stock is given by the following law of motion:

$$
k_{t+1}=\rho\left(k_{t}-x_{t}\right)
$$

Each generation $t$ lives through two periods, youth and old age. It exploits the resource when young as an input to produce consumption units through a production function $f_{t}$. We

\footnotetext{
${ }^{1}$ They might easily end up with more than their aspiration welfare when trading the resource against consumption in competitive markets. In a resource-sharing problem (with an exhaustible resource and a finite number of agents) with a concave and single peak preference similar to that of the present paper, Ambec (2008) shows that the Walrasian allocation with equal division of the resource violates the aspiration upper bounds.
} 
assume that $f_{t}$ is strictly concave and increasing up to a maximal production level $f_{t}\left(\hat{x}_{t}\right)$ and then decreasing. Formally $f_{t}^{\prime}\left(x_{t}\right)>0$ for all $x_{t}<\hat{x}_{t}, f_{t}^{\prime}\left(\hat{x}_{t}\right)=0, f_{t}^{\prime}\left(x_{t}\right)<0$ for all $x_{t}>\hat{x}_{t}$, and $f_{t}^{\prime \prime}\left(x_{t}\right)<0 .^{2}$ The extraction level $\hat{x}_{t}$ is called generation $t$ 's optimal extraction. We also assume that $f_{t}(0)=0$ and $f_{t}^{\prime}(0)=+\infty$ for all $t$. Coexisting generations might perform transfers among themselves. A generation $t$ might share its production when young with old people from the preceding generation. Let $m_{t}$ denote the consumption units transferred by the generation $t$ when young to the generation $t-1$ when old. Thus, generation $t$ consumes $f_{t}\left(x_{t}\right)-m_{t}$ when young and $m_{t+1}$ when old. Without loss of generality $m_{0}$, which denotes the first transfer made by the generation 0 to the generation born in -1 is normalized to zero, since the welfare of generation -1 is not considered here. Let $\gamma_{t}$ be the individual discount rate, i.e. the value in terms of the intertemporal utility at time $t$ of a marginal increase in the instantaneous utility at time $t+1$. We assume that $0<\gamma_{t}<1$. Generation $t$ 's consumption from resource exploitation, hereafter referred to as "utility", viewed at date $t$ with $x_{t}$ units extracted and transfers $m_{t}$ and $m_{t+1}$, is:

$$
u_{t}=f_{t}\left(x_{t}\right)-m_{t}+\gamma_{t} m_{t+1}
$$

We assume that the resource is scarce in the sense that all generations cannot extract enough to meet their demands $\hat{x}_{t}$. More precisely, there exists $\tilde{t} \in \mathbb{N}^{++}$such that if all generations $t<\tilde{t}$ extract $\hat{x}_{t}$, the resource available for generation $\tilde{t}$ is strictly lower than generation $\tilde{t}$ 's optimal extraction $\hat{x}_{\tilde{t}}$. Formally, $\exists \tilde{t}$ such that $\rho^{\tilde{t}} k_{0}-\sum_{t=0}^{\tilde{t}-1} \rho^{\tilde{t}-t} \hat{x}_{t}<\hat{x}_{\tilde{t}}$.

In this set-up with a scarce resource and transferable utility, the selfish outcome under autarky is inefficient (Pareto-dominated) as the following argument shows. Under autarky, it is optimal for each generation $t$ to extract the resource up to $\hat{x}_{t}$. They therefore enjoy $f_{t}\left(\hat{x}_{t}\right)$ consumption units or utility at time $t$. Generation $\tilde{t}$ extracts the remaining resource $\rho^{\tilde{t}} k_{0}-\sum_{t=0}^{\tilde{t}-1} \rho^{\tilde{t}-t} \hat{x}_{t}$, thereby exhausting the resource and leaving nothing for future generations, who therefore obtain $f_{t}(0)$ for all $t>\tilde{t}$. Given that $f_{t}$ is concave with $f_{t}^{\prime}(0)=+\infty$, total production from resource extraction up to a date later than $\tilde{t}$ can be increased if at least one generation $l$ before $\tilde{t}$ reduces its extraction to leave some of the resource for future generations

\footnotetext{
${ }^{2}$ Negative returns above $\hat{x}_{t}$ can be due to production costs that exceed the benefits from resource extraction, e.g. bottleneck effects on complementary inputs (e.g. labor or capital) that render the resource unproductive but nonetheless costly to extract.
} 
after $\tilde{t}$. The increased production can be shared among generations through transfers $m_{t}$ so that every generation becomes better off at least weakly, and strongly for some of them.

We examine coordinated extractions and transfers among generations. Generations agree on an allocation $\left\{x_{t}, m_{t}\right\}_{t=0, \ldots,+\infty}$ that assigns resource extraction levels and intergenerational transfers for every generation $t$. The allocation $\left\{x_{t}, m_{t}\right\}$ must satisfy the following feasibility conditions for all $t \in \mathbb{N}^{+}$:

$$
\begin{aligned}
& 0 \leq x_{t} \leq k_{t} \\
& 0 \leq m_{t} \leq f_{t}\left(x_{t}\right) .
\end{aligned}
$$

The first feasibility condition (3) ensures that the (non-negative) amount of resource extracted does not exceed the stock available at date $t$. The second feasibility condition (4) ensures that the (non-negative) transfer to the old of the previous generation is lower than the consumption goods produced at date $t$.

\section{Core lower bounds}

Our first fairness criterion refers to a fictitious cooperative game. Suppose that all generations can meet to agree on an allocation. A core allocation of the fictitious cooperative game is such that any coalition of generations obtains at least what it could obtain on its own, i.e. by coordinating extraction and carrying out transfers among its members. It satisfies the core lower bound, defined as the highest welfare that a coalition can achieve on its own, for any arbitrary coalition.

In the fictitious cooperative game, generations can share the benefit from resource extraction without constraints: transfers can be carried out among generations that are not contemporaneous in reality. More importantly, non-contemporaneous generations might benefit from coordinated extraction and share this benefit through transfers. In cooperative game theory terms, non-consecutive coalitions can create value. Of course, in the fictitious cooperative game, the sequence of extraction remains fixed: generations cannot exchange the timing of their extraction.

A coalition of generations is a non-empty subset of $\mathbb{N}^{+}$. Given two coalitions $S$ and $T$, we write $S<T$ if $i<j$ for all $i \in S$ and all $j \in T$. Given a coalition $S$, the first and the 
last generation in $S$ are denoted by $\min S$ and $\max S$ respectively. Let $P i=\{1, \ldots, i\}$ denote the set of predecessors of generation $i$, and $P^{0} i=P i \backslash\{i\}$ the set of strict predecessors of generation $i$. Similarly, let $F i=\{i, i+1, \ldots, n\}$ denote the set of followers of generation $i$, and $F^{0} i=F i \backslash\{i\}$ the set of strict followers of generation $i$. We often omit set brackets for sets and write $i$ instead of $\{i\}$, or $v(i, j)$ instead of $v(\{i, j\})$. A coalition $S$ is consecutive (or connected) if for all $i, j \in S$ and all $k \in N, i<k<j$ implies $k \in S$.

We need to define the highest welfare that a coalition can achieve on its own in the fictitious cooperative game. This is a cooperative game with externalities: the welfare of a coalition $S$ depends on extraction strategy by generations outside of $S$ through the stock of resource available to $S$. We assume that the outsiders behave non-cooperatively by extracting the resource under autarky. Consider a coalition $S$. Without loss of generality, let us assign a weight $\theta_{t}$ to agent $t \in S$. The welfare of $S$ is $\sum_{t \in S} \theta_{t} u_{t}$. It means that 1 unit of utility transferred by generation $t$ to generation $t+j$ is valuated at $\theta_{t+j} / \theta_{t}$ units. The value of $\theta_{t}$ is obviously an important issue and, in the rest of this paper, we assume that relative weights equal the generation's discount factor, i.e. $\theta_{t} / \theta_{t-1}=\gamma_{t-1}$. Let us now explain our choice. First, replace the utility of each generation belonging to the coalition in the welfare function to obtain:

$$
\sum_{t \in S} \theta_{t}\left[f_{t}\left(x_{t}\right)-m_{t}+\gamma_{t} m_{t+1}\right]
$$

If the coalition embodies consecutive generations, $S=\{\min S, \ldots, \max S\}$, equation (5) can be rewritten (up to a constant) as follows:

$$
\sum_{t \in S} \frac{\theta_{t}}{\theta_{\min S}} f_{t}\left(x_{t}\right)-m_{\min S}+\sum_{t \in S} \frac{\theta_{t}}{\theta_{\min S}}\left[\gamma_{t}-\frac{\theta_{t+1}}{\theta_{t}}\right] m_{t+1}+\frac{\theta_{\max S}}{\theta_{\min S}} \gamma_{\max S} m_{\max S+1}
$$

Hence, it is only when $\theta_{t} / \theta_{t-1}=\gamma_{t-1}$ that a transfer involving two coexisting generations (i.e. a young individual born at time $t$ and an old one born at time $t-1$ ) is neutral for welfare. If $\theta_{t} / \theta_{t-1}>\gamma_{t-1}$, a transfer from a young individual to an old one has a negative impact on welfare. Transfers are thus likely to be set to zero. Similarly, if $\theta_{t} / \theta_{t-1}<\gamma_{t-1}$, the transfer increases the coalition's welfare. It should then be maximal. When the generations within the coalition are not consecutive, the situation is even worse since the condition $\theta_{t} / \theta_{t-1}=\gamma_{t-1}$ is the only way to make the intergenerational transfer neutral for those who do not belong to the coalition. We thus argue that the welfare of a coalition should discount the utility (or consumption) of future generations because each individual discounts time in her or his own 
utility function. For utility (or consumption) to be transferable without efficiency gain or loss, we need to discount it when we compute the welfare of a coalition. ${ }^{3}$ Under this assumption of neutral transfers, the welfare of coalition $S$ as defined in (5) becomes:

$$
\sum_{t \in S} \Pi_{i=1}^{t} \gamma_{i-1} f_{t}\left(x_{t}\right)
$$

For expositional convenience and without loss of generality, we assume from now on that all generations discount utility at the same rate, $\gamma_{t}=\gamma$, which simplifies coalition $S$ 's welfare as defined in (7) to $\sum_{t \in S} \gamma^{t} f_{t}\left(x_{t}\right){ }^{4}$

Let $v(S)$ be the value function that assigns the highest welfare to any arbitrary coalition $S$. Consider a coalition of consecutive generations $S=\{\min S, \ldots, \max S\}$. The welfare that $S$ can achieve depends on the stock of resource available for the first generation min $S$. We consider the worst possible credible ${ }^{5}$ scenario for $S$, one in which generations preceding the coalition have extracted up to their their optimal level $\hat{x}$ whenever possible. Therefore, the stock of resource, denoted $k_{\min S}^{n c S}$, available for the first generation $\min S$ of a coalition $S$ is:

$$
k_{\min S}^{n c S} \equiv \min \left\{\rho^{\min S} k_{0}-\sum_{t=0}^{\min S-1} \rho^{\min S-t} \hat{x}_{t}, 0\right\} .
$$

Let $x_{S}=\left(x_{i}\right)_{i \in S}$ be the resource allocation assigned to members of $S$. The welfare $v(S)$ valued at date 0 that the consecutive coalition $S$ can achieve on its own is:

$$
\begin{array}{l|l}
v(S)=\max _{x_{S}} \sum_{t \in S} \gamma^{t} f_{t}\left(x_{t}\right), \\
s . t . & \begin{array}{l}
k_{t+1}=\rho\left(k_{t}-x_{t}\right) \\
\\
k_{t} \geq x_{t} \geq 0, k_{t} \geq 0 \\
\\
k_{\min S}=k_{\min S}^{n c S} .
\end{array}
\end{array}
$$

\footnotetext{
${ }^{3} \mathrm{~A}$ consistent explanation for both individual and social discounting relies on the possibility, at each period of time, of the end of the world (Dasgupta and Heal, 1974 and 1979). The discount rate is then the world's survival probability and, provided that the utility is zero in the case of a collapse, objectives (2) and (5) can be seen as expected utility functions. Equalizing the individual and coalition discount rates would, in this case, be reasonable.

${ }^{4}$ This assumption is not needed for the proof of Proposition 1, which therefore holds for heterogenous discount rates.

${ }^{5}$ Extracting more than $\hat{x}_{t}$ is not credible for a generation $t$ since it reduces production and therefore utility.
} 
The constraints on the maximization program are the resource dynamics, the feasibility and the initial resource stock conditions respectively. In particular, for singletons $S=\{i\}$, we have

$$
v(i)=f_{i}\left(\min \left\{\hat{x}_{i}, k_{i}^{n c}\right\}\right) .
$$

For any arbitrary coalition $S$, let $\mathcal{C}(S)=\left\{T_{l}\right\}_{l=1}^{L}$, where $T_{1}<T_{2}<\ldots<T_{L}$ denotes the set of connected components of $S$, i.e. $\mathcal{C}(S)$ is the coarsest partition of $S$ such that any $T_{l} \in \mathcal{C}(S)$ is connected. Since the generations between two consecutive sub-coalitions $T_{l-1}, T_{l} \in \mathcal{C}(S)$ extract up to their optimal level $\hat{x}$, given the resource stock $k_{\max T_{l}}$ left by the last generation in $T_{l}$, the resource stock available for $T_{l}$ for $l=2, \ldots, L$ is

$$
k_{\min T_{l}}^{n c S} \equiv \min \left\{\rho^{\left(\min T_{l}-\max T_{l-1}+1\right)} k_{\max T_{l-1}+1}-\sum_{t=\max T_{l-1}+1}^{\min T_{l}-1} \rho^{\left(\min T_{l}-t\right)} \hat{x}_{t}, 0\right\} .
$$

The welfare $v(S)$ valued at date 0 that $S$ can achieve on its own is thus:

$$
\begin{array}{l|l}
v(S)=\max _{x_{S}} \sum_{t \in S} \gamma^{t} f_{t}\left(x_{t}\right), \\
\text { s.t. } & k_{t+1}=\rho\left(k_{t}-x_{t}\right), \\
& k_{t} \geq x_{t} \geq 0, k_{t} \geq 0, \\
& k_{\min T_{l}}=k_{\min T_{l}}^{n c S} \text { for } l=1, \ldots, L
\end{array}
$$

In contrast to the case of consecutive coalitions, the initial resource stock constraints are defined for each consecutive component of $S$. Let $x_{S}^{S}$ denote the solution to (9) for any coalition $S$.

An important property of the value function defined in (9) is its superadditivity. Consider any disjoint coalitions $T, S \subset \mathbb{N}^{+}$. Since the resource allocation $\left(x_{T}^{T}, x_{S}^{S}\right)$ can be implemented by coalition $T \cup S$, we have:

$$
v(S \cup T) \geq v(S)+v(T)
$$

An allocation $\left\{x_{t}, m_{t}\right\}$ satisfies the core lower bounds if and only if for all coalitions $S \subset \mathbb{N}^{+}$

$$
\sum_{t \in S} \gamma^{t}\left(f_{t}\left(x_{t}\right)-m_{t}+\gamma m_{t+1}\right) \geq v(S)
$$




\section{Aspiration upper bounds}

Our second criterion is based on a solidarity principle inspired by Moulin (1990). In the absence of other generations, a generation $t$ would be endowed with $\rho^{t} k_{0}$ units of the resource, which is the "natural" stock. It could enjoy the benefit of extracting this resource stock up to its optimal level $\hat{x}_{t}$. Let us call this benefit valued at date 0 the generation $t$ 's aspiration welfare, and let it be denoted by $W(t)=\gamma^{t} f_{t}\left(\min \left\{\rho^{t} k_{0}, \hat{x}_{t}\right\}\right)$. Since the resource is scarce in the precise sense that $\rho^{t} k_{0}-\sum_{j=0}^{t-1} \rho^{j-t} \hat{x}_{j}<\hat{x}_{t}$ for all $t \geq \tilde{t}$, it is impossible for every generation to be assigned its aspiration welfare. ${ }^{6}$ In Moulin (1990)'s terms, the sustainable resource exploitation problem exhibits negative group externalities. Because no particular generation bears any distinct responsibility for these externalities, it is only natural to ask for every generation to accept a share of it: no generation should end up above its aspiration welfare. This argument can be generalized to coalitions in a very natural way. The aspiration welfare of an arbitrary coalition $S$ is the highest welfare it could achieve in the absence of other generations. ${ }^{7}$

In contrast to the core lower bounds $v(S)$, coalition $S$ inherits from an untouched resource when the aspiration welfare is computed. Formally, coalition $S$ has access to $\rho^{\min S} k_{0}>k_{\min S}^{n c S}$. For connected coalitions, it is the solution to the following program:

$$
\begin{array}{l|l}
w(S)=\max _{x_{S}} \sum_{t \in S} \gamma^{t} f_{t}\left(x_{t}\right), \\
s . t . & \begin{array}{l}
k_{t+1}=\rho\left(k_{t}-x_{t}\right), \\
k_{t} \geq x_{t} \geq 0, k_{t} \geq 0, \\
\\
k_{\min S}=\rho^{\min S} k_{0} .
\end{array}
\end{array}
$$

The constraints in the maximization program are the resource dynamics, the feasibility and the initial resource stock respectively.

A disconnected coalition $S$ that leaves some resource stock after the last generation in $T_{l}$ to supply the generations in $T_{l+1}$ experiences no extraction from outsiders. Therefore, the

\footnotetext{
${ }^{6}$ Indeed, for any $t>\tilde{t}$ (where $\tilde{t}$ is defined above) and consecutive coalitions $t \in S$, we have $\sum_{t \in S} w(t)>$ $v(S)$, that is, the sum of the generations' aspiration welfare exceeds the maximal welfare from resource exploitation.

${ }^{7}$ Similar to the case of the core lower bounds, we allow for transfers among non-contemporaneous generations in $S$.
} 
resource stock entering $T_{l+1}$ is $\rho^{\left(\min T_{l+1}-\max T_{l}\right)} k_{\max T_{l}}$. The aspiration welfare of an arbitrary coalition $S$ is thus:

$$
\begin{array}{l|l}
w(S)=\max _{x_{S}} \sum_{t \in S} \gamma^{t} f_{t}\left(x_{t}\right), \\
\text { s.t. } & \begin{array}{l}
k_{t+1}=\rho\left(k_{t}-x_{t}\right), \\
k_{t} \geq x_{t} \geq 0, k_{t} \geq 0, \\
k_{\min T_{l}}=\rho^{\left(\min T_{l+1}-\max T_{l}\right)} k_{\max T_{l}} \text { for } l=1, \ldots, L .
\end{array}
\end{array}
$$

The constraints in the maximization program are the resource dynamics, the feasibility and the initial resource stock conditions respectively. The main difference between programs (9) and (12) lies in the initial resource stocks that are reduced by generations outside of $S$ in (9) but not in (12).

An allocation $\left\{x_{t}, m_{t}\right\}$ satisfies the aspiration upper bounds if and only if, for all coalitions $S \subset \mathbb{N}^{+}$

$$
\sum_{t \in S} \gamma^{t}\left(f_{t}\left(x_{t}\right)-m_{t}+\gamma m_{t+1}\right) \leq w(S)
$$

\section{A unique fair allocation}

Consider the efficient resource allocation $\left\{x_{t}^{*}\right\}$ solution to the maximization program defined by $v\left(\mathbb{N}^{+}\right)$. Formally, $\left\{x_{t}^{*}\right\}$ maximizes $\sum_{t=0}^{\infty} \gamma^{t} f_{t}\left(x_{t}\right)$ subject to the initial resource stock constraint $k_{0}$, the resource dynamics constraint $k_{t+1}=\rho\left(k_{t}-x_{t}\right)$ and the feasibility constraints $k_{t} \geq x_{t} \geq 0$ for $t=0,1,2, \ldots$. The concavity of $f_{t}$ ensures that $\left\{x_{t}^{*}\right\}$ is unique.

A transfer scheme $\left\{m_{t}\right\}$ defines a distribution of the welfare from intergenerational resource extraction. We focus on the transfer scheme that leads to the downstream welfare distribution introduced by Ambec and Sprumont (2002). Denoted by $\left\{m_{t}^{*}\right\}$, it is the unique transfer scheme in which each generation is assigned its marginal contribution to the preceding generation. Formally, $\left\{x_{t}^{*}, m_{t}^{*}\right\}$ assigns $u_{t}^{*}=f_{t}\left(x_{t}^{*}\right)-m_{t}^{*}+\gamma m_{t+1}^{*}$ to every generation $t \in \mathbb{N}^{+}$with:

$$
\gamma^{t} u_{t}^{*}=v(P t)-v\left(P^{0} t\right)
$$

Proposition 1 If $m_{t}^{*} \leq f_{t}\left(x_{t}^{*}\right)$ for all $t \in \mathbb{N}^{+},\left\{x_{t}^{*}, m_{t}^{*}\right\}$ is the unique allocation that satisfies the core lower bounds and the aspiration upper bounds. 


\section{Proof}

First, we prove that if an allocation $\left\{x_{t}\right\}$ satisfies the core lower bounds $\left\{x_{t}\right\}=\left\{x_{t}^{*}\right\}$. The core lower bounds imply that:

$$
\sum_{t=0}^{j} \gamma^{t}\left(f_{t}\left(x_{t}\right)-m_{t}+\gamma m_{t+1}\right) \geq v(P j),
$$

for all $j \in \mathbb{N}^{+}$. Since $\sum_{t=0}^{j} \gamma^{t}\left(f_{t}\left(x_{t}\right)-m_{t}+\gamma m_{t+1}\right)$, the above inequality for $j \longrightarrow \infty$ leads to

$$
\sum_{t=0}^{\infty} \gamma^{t} f_{t}\left(x_{t}\right)+\lim _{j \longrightarrow \infty} \gamma^{j+1} m_{j+1} \geq v\left(\mathbb{N}^{+}\right)
$$

Since $\gamma<1$ then $\lim _{j \longrightarrow \infty} \gamma^{j+1}=0$ and, since the feasibility constraint (4) upper bounds $m_{j+1}, \lim _{j \longrightarrow \infty} \gamma^{j+1} m_{j+1}=0$. Therefore (14) implies

$$
\sum_{t=0}^{\infty} \gamma^{t} f_{t}\left(x_{t}\right) \geq v\left(\mathbb{N}^{+}\right)
$$

which, when combined with the definition of $v\left(\mathbb{N}^{+}\right)$, implies that $\left\{x_{t}\right\}=\left\{x_{t}^{*}\right\}$.

Second, it is easy to see that if a welfare distribution $\left\{m_{t}\right\}$ satisfies both the core lower bounds and the aspiration upper bounds, then $\left\{m_{t}\right\}=\left\{m_{t}^{*}\right\}$. This is due to the fact that for coalitions starting from 0 up to any generation $t$, we have $v(P t)=w(P t) .{ }^{8}$ Given $m_{0}$, since $v(0)=w(0)$, we must have $m_{1}=m_{1}^{*}$. Let $m_{t}=m_{t}^{*}$ for all $t \leq j+1$. The core constraints and the aspiration upper bounds force $\sum_{t=0}^{j} \gamma^{t}\left(f_{t}\left(x_{t}^{*}\right)-m_{t}+\gamma m_{t+1}\right)=v(P j)$, hence $\gamma^{j}\left(f_{j}\left(x_{j}^{*}\right)-m_{j}+\gamma m_{j+1}\right)=v(P j)-\sum_{t=0}^{j-1} \gamma^{t}\left(f_{t}\left(x_{t}^{*}\right)-m_{t}+\gamma m_{t+1}\right)$. Thus by $m_{t}=m_{t}^{*}$ for all $t \leq j+1$, then $\sum_{t=0}^{j-1} \gamma^{t}\left(f_{t}\left(x_{t}^{*}\right)-m_{t}+\gamma m_{t+1}\right)=\sum_{t=0}^{j-1} \gamma^{t}\left(f_{t}\left(x_{t}^{*}\right)-m_{t}^{*}+\gamma m_{t+1}^{*}\right)=v\left(P^{0} j\right)$, we therefore obtain $\gamma^{j}\left(f_{j}\left(x_{j}^{*}\right)-m_{j}+\gamma m_{j+1}\right)=v(P j)-v\left(P^{0} j\right)$, i.e. the desired conclusion.

Next, we show that $\left\{x_{t}^{*}, m_{t}^{*}\right\}$ satisfies the core lower bounds, that is, $\sum_{t \in S} \gamma^{t} u_{t}^{*} \geq v(S)$ for any coalition $S$ where $u_{t}^{*} \equiv f_{t}\left(x_{t}^{*}\right)-m_{t}^{*}+\gamma m_{t+1}^{*}$.

Before we proceed, we note the following: for all $t$, we have $v\left(P^{0} t\right)+\gamma^{t} f_{t}\left(\hat{x}_{t}\right) \geq v(P t)$. Thus for all generations $t$,

$$
\gamma^{t} f_{t}\left(\hat{x}_{t}\right) \geq v(P t)-v\left(P^{0} t\right)
$$

\footnotetext{
${ }^{8}$ Note that the uniqueness of our solution is due the equality of the core lower bounds and aspiration welfare upper bounds for all consecutive coalitions starting from 0, i.e. $v(P t)=w(P t)$ for all $t$. Notably, if we bound the size of coalitions that might be formed to say $n \ll+\infty$, other transfer schemes might satisfy the two fairness axioms. More precisely, $m_{t}$ can differ from $m_{t}^{*}$ for generations $t>n$.
} 
Suppose first of all that $S$ is a consecutive coalition. Since $P S=P^{0} S \cup S$, by superadditivity of $v, v(P S) \geq v\left(P^{0} S\right)+v(S)$ and $\sum_{t \in S} \gamma^{t} u_{t}^{*}=v(P S)-v\left(P^{0} S\right)$, which implies that $\sum_{t \in S} \gamma^{t} u_{t}^{*} \geq v(S)$.

Second, consider any coalition $S$. Take the last generation in $S$ that obtains some resource $l(S)=\max _{t}\left\{t \in S: x_{t}^{S}>0\right\}$. If $l(S)$ does not exist then $v(S)=0 \leq \sum_{t \in S} \gamma^{t} u_{t}^{*}$. Let $\bar{S}=P l(S) \backslash P^{0} \min S$ be the coalition of all generations from $\min S$ to $l(S)$. Since $\bar{S}$ is connected, $\sum_{t \in \bar{S}} \gamma^{t} u_{t}^{*}=v(P \bar{S})-v\left(P^{0} \bar{S}\right) \geq v(\bar{S})$. Adding $\sum_{t \in \bar{S} \backslash S} \gamma^{t} u_{t}^{*}$ to both sides of the last inequality yields:

$$
\sum_{t \in S} \gamma^{t} u_{t}^{*} \geq v(\bar{S})-\sum_{t \in \bar{S} \backslash S} \gamma^{t} u_{t}^{*}
$$

Since generations between connected coalitions in $S$ up to $l(S)$ extract up to their optimal level, the allocation $\left(x_{S \cap P l(S)}^{S}, \hat{x}_{S \backslash \bar{S}}\right)$ can be implemented in $\bar{S}$, which implies

$$
v(\bar{S}) \geq v(S \cap P l(S))+\sum_{t \in \bar{S} \backslash S} f_{t}\left(\hat{x}_{t}\right) .
$$

Since there is no more resource to be shared in $S$ after $l(S), f_{t}\left(x_{t}^{S}\right)=f_{t}(0)=0$ for any $t \in S \backslash P l(S)$, which therefore implies that $v(S)=v(S \cap P l(S))$. We combine (16) and (17) to obtain

$$
\sum_{t \in S} \gamma^{t} u_{t}^{*} \geq v(S)+\sum_{t \in \bar{S} \backslash S} \gamma^{t}\left(f_{t}\left(\hat{x}_{t}\right)-u_{t}^{*}\right)
$$

From (15), we know that $\gamma^{t} f_{t}\left(\hat{x}_{t}\right) \geq \gamma^{t} u_{t}^{*}$ for all $t$. Hence, $\sum_{t \in S} \gamma^{t} u_{t}^{*} \geq v(S)$, which shows that $\left\{m_{t}^{*}\right\}$ satisfies the core lower bounds.

Lastly, we show that $\left\{x_{t}^{*}, m_{t}^{*}\right\}$ satisfies the aspiration upper bounds. The proof uses the following lemma that is proven in the Appendix.

Lemma 1 If $S \subseteq T \subseteq N$ and $T<i$, then $w(S \cup i)-w(S) \geq w(T \cup i)-w(T)$.

Then for any coalition $S$, we obtain

$$
\sum_{i \in S} \gamma^{t} u_{i}^{*}=\sum_{i \in S}\left(w(P i)-w\left(P^{0} i\right)\right) \leq \sum_{i \in S}\left(w(P i \cap S)-w\left(P^{0} i \cap S\right)\right)=w(S)
$$

where the inequality follows from Lemma 1 and the latter equality follows from the fact that all terms, except $w(P \max S \cap S)=w(S)$ and $-w\left(P^{0} \min S \cap S\right)=w(\emptyset)=0$,cancel out. 


\section{Description of fair allocation}

Let us now describe the unique allocation, denoted by $\left\{x_{t}^{*}, m_{t}^{*}\right\}$, which satisfies the core lower bounds and the aspiration upper bounds. To proceed, we need some additional assumptions on the time dependency of the production function. We will notably focus on the time-invariant case such that $f_{t}(x)=f_{t+1}(x)$, which can be interpreted as the case with no technical progress. We will then provide some intuitions on how the fair allocation is modified when some specific technical progress is introduced.

Proposition 1 states that the fair path of extraction $\left\{x_{t}^{*}\right\}$ is an efficient one. It can therefore be studied independently of the fair path of transfers $\left\{m_{t}^{*}\right\}$. In the specific case where $f_{t}(x)=f_{t+1}(x)$, which implies that $\hat{x}_{t}=\hat{x}_{t+1},\left\{x_{t}^{*}\right\}$ is the solution of the following problem:

$$
\begin{aligned}
& \max _{\left\{x_{t}\right\}} \sum_{t=0}^{\infty} \gamma^{t} f\left(x_{t}\right), \\
& \begin{array}{l|l} 
& k_{t+1}=\rho\left(k_{t}-x_{t}\right), \\
\text { s.t. } & x_{t} \geq 0, k_{t} \geq 0, \\
& k_{0}>0 \text { given. }
\end{array}
\end{aligned}
$$

The following Proposition characterizes the solution to problem (18).

Proposition 2 If $f_{t}(x)=f(x)$ for all $t$, the fair path of extraction $\left\{x_{t}^{*}\right\}$ and the stock of resource are:

i) monotonically increasing if $\gamma \rho>1$ with an asymptotic constant extraction path $x_{\infty}^{*}=\hat{x}$ and $k_{\infty}=\frac{\rho}{\rho-1} \hat{x}$

ii) monotonically decreasing if $\gamma \rho<1$ with a stock that is asymptotically exhausted,

iii) constant for all $t$ if $\gamma \rho=1$ with a constant extraction path $x_{t}^{*}=\left(1-\frac{1}{\rho}\right) k_{0}$ for all $t$.

\section{Proof}

To begin, let us observe that an $x_{t}$ is optimal if and only if it belongs to $[0, \hat{x}]$. Suppose by contradiction that $\tilde{x}_{t}$ is optimal and is such that $\tilde{x}_{t}>\hat{x}$. Then, there exists $\varepsilon>0$ such that $f\left((1-\varepsilon) \tilde{x}_{t}\right)>f\left(\tilde{x}_{t}\right)$ and $\rho\left(k_{t}-(1-\varepsilon) x_{t}\right)>\rho\left(k_{t}-\tilde{x}_{t}\right)$. Hence $\tilde{x}_{t}$ is not optimal. 
The first order condition of problem (18) is:

$$
f^{\prime}\left(x_{t-1}\right)-\gamma \rho f^{\prime}\left(x_{t}\right)=0
$$

for all $t \in \mathbb{N}^{++}$, while the transversality condition is:

$$
\lim _{t \rightarrow+\infty} \gamma^{t} f^{\prime}\left(x_{t}\right) k_{t+1}=0
$$

Hence $\left\{x_{t}^{*}\right\}$ solves (19), the resource constraint and (20). Since $x_{t} \geq x_{t-1} \Leftrightarrow f^{\prime}\left(x_{t}\right) \leq f^{\prime}\left(x_{t-1}\right)$, we use (19) to conclude that: $x_{t}^{*} \geq x_{t-1}^{*} \Leftrightarrow \gamma \rho \geq 1$. Thus, there are three distinct cases depending on the value of $\gamma \rho$.

Case 1: $\gamma \rho>1$. The optimal trajectory $x_{t}^{*}$ converge to $\hat{x}$. It remains to determine $x_{0}^{*}$. There are three families of candidates that are represented in the following phase diagram (see Figure $1)$.

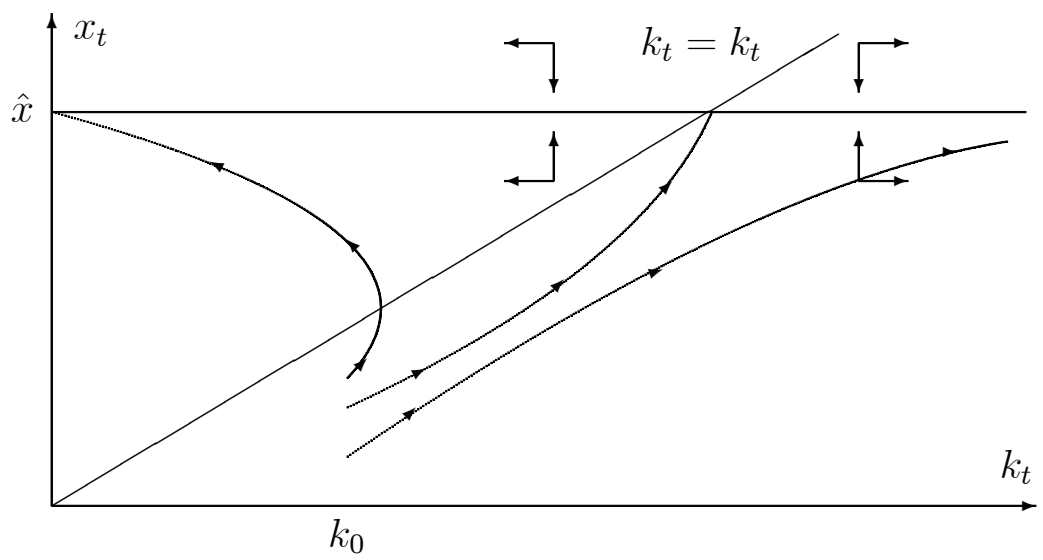

Figure 1

The first family of candidates is such that $k_{t}$ converges to 0 . After a while, this convergence is monotonic. With equation $k_{t+1}=\rho\left(k_{t}-x_{t}\right)$, this implies that $x_{t}$ converges to 0 , which is a contradiction. These trajectories are not optimal. The second family of candidates is such that $k_{t}$ converge to $+\infty$. These trajectories do not satisfy the transversality condition. Indeed, on the optimal path, one has:

$$
\frac{\gamma^{t} f^{\prime}\left(x_{t}\right) k_{t+1}}{\gamma^{t-1} f^{\prime}\left(x_{t-1}\right) k_{t}}=\frac{k_{t+1}}{\rho k_{t}}=1-\frac{x_{t}}{k_{t}}
$$

where the first equality comes from (19) and the second from the resource constraint. Therefore,

$$
\lim _{t \rightarrow+\infty} \frac{\gamma^{t} f^{\prime}\left(x_{t}\right) k_{t+1}}{\gamma^{t-1} f^{\prime}\left(x_{t-1}\right) k_{t}}=1 \text { and } \lim _{t \rightarrow+\infty} \gamma^{t} f^{\prime}\left(x_{t}\right) k_{t+1} \rightarrow+\infty
$$


The third candidate is the saddle point solution for which $k_{t}$ converges to $\frac{\rho}{\rho-1} \hat{x}$. This solution satisfies the transversality condition. Along the trajectory, the resource stock is monotonically increasing.

Case 2: $\gamma \rho<1$. Because of the condition $\lim _{x \rightarrow 0} f^{\prime}(x)=+\infty$, the optimal trajectory $x_{t}^{*}$ converges to 0 . To determine $x_{0}^{*}$, one should study two families of candidates that are represented in the following phase diagram (see Figure 2).

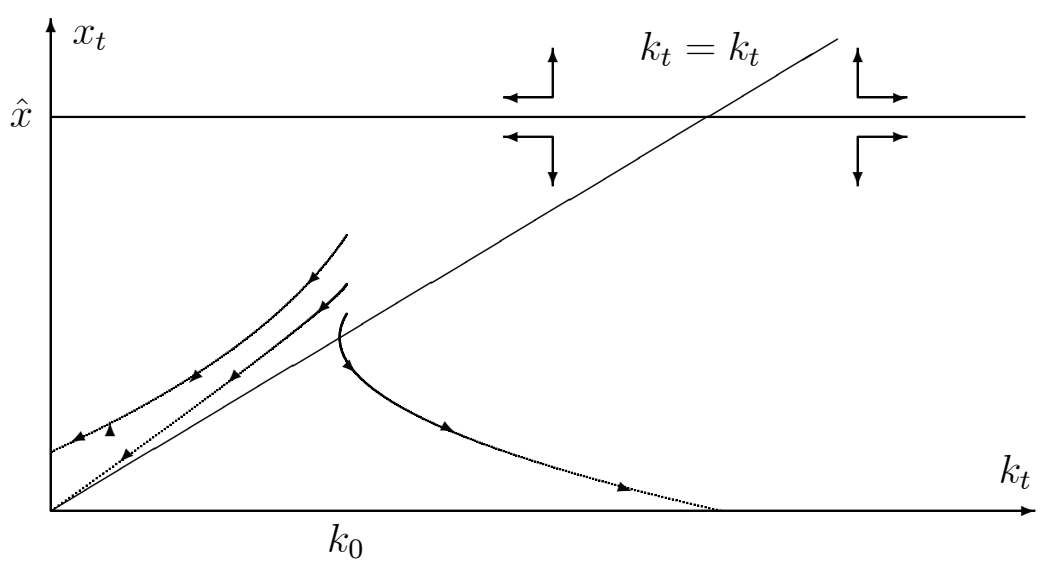

Figure 2

The first family of candidates is such that $k_{t}$ converges to 0 . Among them, only one is such that $x_{t}^{*}$ converges to 0 , while the others exhibit a sequence of $x_{t}$ that converges to positive values, which is thus impossible. It remains for us to check that the good trajectory satisfies the transversality condition. On the optimal path, since $k_{t}$ converge to 0 , one has:

$$
\frac{\gamma^{t} f^{\prime}\left(x_{t}\right) k_{t+1}}{\gamma^{t-1} f^{\prime}\left(x_{t-1}\right) k_{t}}=\frac{k_{t+1}}{\rho k_{t}}<\frac{1}{\rho}<1
$$

from which we deduce that: $\lim _{t \rightarrow+\infty} \gamma^{t} f^{\prime}\left(x_{t}\right) k_{t+1}=0$. Along this path, the stock of the resource decreases monotonically and is asymptotically exhausted.

The second family of candidates is such that $k_{t}$ converges to $+\infty$. As in Case 1 , these trajectories do not satisfy the transversality condition.

Case 3: $\gamma \rho=1$. In this particular case, any constant solution solves (19). Let $x^{*}$ be the optimal solution. Given the objective: $\max _{x t} \sum_{t=0}^{\infty} \gamma^{t} f\left(x_{t}\right)$, the closer $x^{*}$ is to $\hat{x}$, the better. To compute $x^{*}$, we rewrite the resources dynamics such that:

$$
k_{t+1}=\rho^{t+1}\left[k_{0}-x^{*} \frac{1-\gamma^{t+1}}{1-\gamma}\right]
$$

and substitute this expression into (20) to obtain: $\lim _{t \rightarrow+\infty} f^{\prime}\left(x^{*}\right) \rho\left[k_{0}-x^{*} /(1-\gamma)\right]=0$. The optimal solution is thus: $x^{*}=(1-\gamma) k_{0}$ if $(1-\gamma) k_{0}<\hat{x}$, and $x^{*}=\hat{x}$ otherwise. The 
latter solution is eliminated on the assumption of resource scarcity. In the former, the stock of resource is constant.

We note that these results can be immediately extended to specific technical progress. Let us suppose for instance that: $f_{t}\left(x_{t}\right)=A_{t} f\left(x_{t}\right)=A_{0} \eta^{t} f($.$) with 1 \leq \eta<1 / \gamma$. The problem can now be written as: $\max _{x t} \sum_{t=0}^{\infty}(\gamma \eta)^{t} f\left(x_{t}\right)$, subject to the same constraint. The problem is thus the same as (18), apart from the fact that we now compare $\gamma \eta$ with $\rho$.

Another way to introduce technical progress would be to suppose that $f_{t}\left(x_{t}\right)=f\left(A_{t} x_{t}\right)$ with $A_{t}=A_{0} \eta^{t}$ and $\eta \geq 1$. The first order condition (19) should then be replaced by: $f^{\prime}\left(A_{t-1} x_{t-1}\right)-\gamma \rho \eta f^{\prime}\left(A_{t} x_{t}\right)=0$. Defining: $\breve{x}_{t}=A_{t} x_{t}$ and $\breve{k}_{t}=A_{t} k_{t}$, the optimal solution can thus be found by solving:

$$
\left\{\begin{array}{c}
\breve{k}_{t+1}-\rho \eta\left(\breve{k}_{t}-\breve{x}_{t}\right)=0 \\
f^{\prime}\left(\breve{x}_{t-1}\right)-\gamma \rho \eta f^{\prime}\left(\breve{x}_{t}\right)=0
\end{array}\right.
$$

which is the same as the one studied previously, provided that $\rho$ is replaced by $\rho \eta$.

Let us now turn to the characterization of the fair path of transfers $\left\{m_{t}^{*}\right\}$. From Proposition 1, we have:

$$
m_{t+1}^{*}=\frac{\sum_{i=0}^{t} \gamma^{i} f_{i}\left(x_{i}^{P_{t}}\right)-\sum_{i=0}^{t} \gamma^{i} f_{i}\left(x_{i}^{*}\right)}{\gamma^{t+1}}
$$

for all $t \in \mathbb{N}^{+}$, and where $x_{i}^{P_{t}}$ is the solution to $\max _{x_{i}} \sum_{i=0}^{t} \gamma^{i} f_{i}\left(x_{i}\right)$ subject to the resource and non-negativity constraints. As it has been discussed above, $\lim _{t \rightarrow+\infty} x_{i}^{P_{t}}=x_{i}^{*}$. Hence, by the definition of the maximum, $m_{t+1}^{*} \geq 0$. However, we have seen that fair allocation exists if and only if $m_{t+1}^{*} \leq f_{t+1}\left(x_{t+1}^{*}\right)$ for all $t \in \mathbb{N}^{+}$. We would like to stress that this condition is very restrictive and is not satisfied in many cases. Indeed, fair transfers are likely to increase over time: each generation has to compensate the previous one for not exploiting the resource in an autarkic way and also for having compensated the previous generation. Hence, as shown in the following Lemma, fair transfers increase, at least for an initial interval of time.

Proposition 3 Fair transfers satisfy: $m_{t+2}^{*} \geq m_{t+1}^{*}$, for all $t \leq \tilde{t}-2$, 


\section{Proof}

Using (21), $m_{t+2}^{*} \geq m_{t+1}^{*}$ if and only if:

$$
\sum_{i=0}^{t} \gamma^{i}\left[f_{i}\left(x_{i}^{P_{t+1}}\right)-\gamma f_{i}\left(x_{i}^{P_{t}}\right)\right]+\gamma^{t+1} f_{t+1}\left(x_{t+1}^{P_{t+1}}\right) \geq(1-\gamma) \sum_{i=0}^{t} \gamma^{i} f_{i}\left(x_{i}^{*}\right)+\gamma^{t+1} f_{t+1}\left(x_{t+1}^{*}\right)
$$

We recall that $\tilde{t}$ relies on the scarcity of the resource and gives the date at which the resource is depleted under autarky. Hence, for all $t \leq \tilde{t}-2$, the resource is abundant and the optimal exploitation is kept at the generations' optimal extraction: i.e. $x_{i}^{P_{t+1}}=\hat{x}_{i}$. The previous inequality can hence be rewritten as:

$$
(1-\gamma) \sum_{i=0}^{t} \gamma^{i} f_{i}\left(\hat{x}_{i}\right)+\gamma^{t+1} f_{t+1}\left(\hat{x}_{t+1}\right) \geq(1-\gamma) \sum_{i=0}^{t} \gamma^{i} f_{i}\left(x_{i}^{*}\right)+\gamma^{t+1} f_{t+1}\left(x_{t+1}^{*}\right),
$$

which, given that $\hat{x}_{t} \geq x_{t}^{*}$ for all $t$ from Proposition 2 , is obviously satisfied.

Let us illustrate the existence problem driven by the increase of transfers over time by a simple numerical application. Using Proposition 2, a specific case can indeed be easily derived. Suppose that $\gamma \rho=1$, and that $f_{t}\left(x_{t}\right)=\sqrt{x_{t}}$ for $x_{t} \leq \hat{x}$, where the value of $\hat{x}$ is sufficiently high (e.g. $\left.\hat{x}=k_{0}\right){ }^{9}$ Thus, $x_{i}^{P_{t}}=(1-\gamma) k_{0} /\left(1-\gamma^{t+1}\right)$ for all $i$, and:

$$
m_{t+1}^{*}=\frac{\sqrt{\frac{k_{0}}{(1-\gamma)}}\left(\sqrt{1-\gamma^{t+1}}-\left(1-\gamma^{t+1}\right)\right)}{\gamma^{t+1}}
$$

which can be shown to be an increasing function of time. Moreover, since $x_{i}^{*}=(1-\gamma) k_{0}$ for all $i$, the feasibility condition $m_{t+1}^{*} \leq f_{t+1}\left(x_{t+1}^{*}\right)$ is rewritten as: $\sqrt{1-\gamma^{t+1}} \leq\left(1-\gamma^{t+2}\right)$, which is always satisfied for low enough $\gamma$ and never satisfied for large enough $\gamma$. For instance, $m_{t+1}^{*}$ and $f_{t+1}\left(x_{t+1}^{*}\right)$ are plotted as (continuous) functions of time in the figures below for various values of $\gamma$. The increasing dashed blue curve represents $m_{t+1}^{*}$ while the solid red line is the constant $f_{t+1}\left(x_{t+1}^{*}\right)$. We see that the condition is satisfied for $\gamma=0.3$ and $\gamma=0.5$, while it is not for $\gamma=0.7$. To interpret this, let us recall that a larger $\gamma$ implies (in this very specific

\footnotetext{
${ }^{9}$ More precisely, we need $\hat{x}_{t}>x_{i}^{*}=(1-\gamma) k_{0}$.
} 
case) a lower resource regeneration rate.
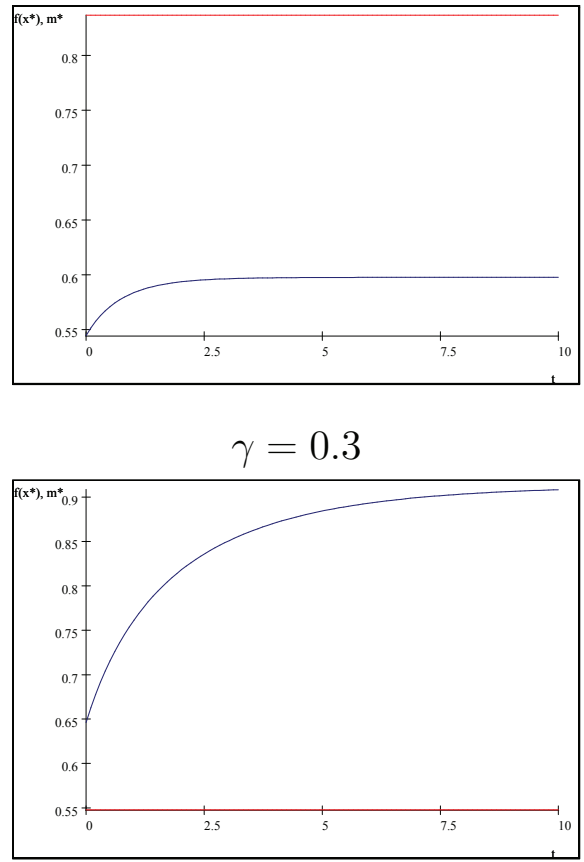

$$
\gamma=0.7
$$

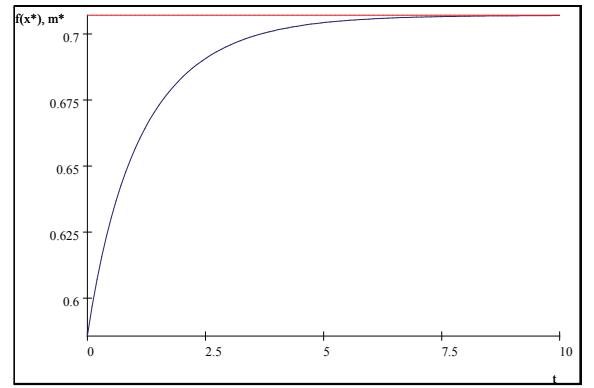

$\gamma=0.5$

Figures 3

It is worth mentioning that for $\gamma=0.7$, the feasibility condition is violated "from the beginning", that is, for the first transfer $m_{1}$ between the first two generations 1 and 2 . More precisely, along the efficient extraction path, the second generation has not enough production to transfer to the first generation to make it as well off as it would be under autarky. Indeed, in this example, it is impossible to find a compensation scheme that satisfies the core lower bounds for coalitions composed by two successive generations only. Therefore, relaxing the core lower bounds by allowing coalitions of limited size to form does not guarantee existence.

To conclude this characterization of fair allocation let us discuss the dynamics of the utilities of each generation $u_{t}^{*}$. The following Proposition gives a sufficient condition under which the utilities decrease over time.

Proposition 4 For all $t \geq 2, u_{t}^{*} \leq u_{1}^{*}$ if $f_{t}\left(x_{t}^{P_{t}}\right) \leq f_{1}\left(\hat{x}_{1}\right)$.

\section{Proof}

The Proof of Proposition 1 implies that $u_{1}^{*}=f_{1}\left(\hat{x}_{1}\right)$. As a consequence, $u_{t}^{*} \leq u_{1}^{*} \Leftrightarrow$ 
$\gamma m_{t+1}^{*}-m_{t} \leq f_{1}\left(\hat{x}_{1}\right)-f_{t}\left(x_{t}^{*}\right)$, which using (21) implies that $u_{t}^{*} \leq u_{1}^{*}$ if and only if:

$$
\sum_{i=0}^{t-1} \gamma^{i} f_{i}\left(x_{i}^{P_{t}}\right)-\sum_{i=0}^{t-1} \gamma^{i} f_{i}\left(x_{i}^{P_{t-1}}\right) \leq \gamma^{t}\left[f_{1}\left(\hat{x}_{1}\right)-f_{t}\left(x_{t}^{P_{t}}\right)\right] .
$$

Using the definition of a maximum, we observe that the left-hand side of the inequality is negative, which is sufficient for us to conclude.

A direct implication of this is that technical progress is a necessary condition for fair allocation to keep the utilities at least constant. Indeed, if $f_{t}(x)=f_{t+1}(x)$, then the maximal production level decided by the first generation cannot be overcome.

\section{Conclusion}

In this paper, we proposed a fair allocation of a scarce resource over an infinite sequence of overlapping generations. When it satisfies two fairness criteria, namely the core lower bounds and the aspiration upper bounds, the allocation is unique. The exploitation of the resource is efficient and there is no generation left without any resource. First generations are compensated through a transfer scheme in which each generation is assigned its marginal contribution to the preceding generation. Such a scheme is likely to induce an increase in transfers over time that may cause the infeasibility of the allocation. Finally, technical progress is necessary for avoiding the decrease of the utilities of future generations. One remaining issue is the stability of the fair allocation, which is related to our last result. If utilities decrease over time while the resource stock increases, future generations have an incentive to deviate. This important question has been left for future research. 


\section{A Proof of Lemma 1}

This proof is adapted from Ambec and Ehlers (2008). Let $y_{S}^{S}$ denote the solution of the program defined by $w(S)$ in (12) for any arbitrary coalition $S \subset \mathbb{N}^{+}$. As a first step in the proof of this lemma, let us show that if $\emptyset \neq S \subset T \subset N$, then $y_{S}^{S} \geq y_{S}^{T}$. Clearly, it suffices to establish that $y_{S}^{S} \geq y_{S}^{S \cup t}$ whenever $\emptyset \neq S \neq N$ and $t \in N \backslash S$. Let us write $y_{S}^{S}=x_{S}$ and $y_{S}^{S \cup t}=y_{S}$. All agents under consideration in the argument belong to $S$. From the definition of $x$ and $y, \sum_{i \in S} y_{i} \leq \sum_{i \in S} x_{i}$. Let $i_{1} \leq \ldots \leq i_{L}$ be those $i$ such that $x_{i} \neq y_{i}$ (if none exists, there is nothing to prove). We claim that $y_{i_{1}}<x_{i_{1}}$. Suppose, by contradiction, that the opposite (necessarily strict) inequality is true. Let $j$ be the smallest successor of $i_{1}$ such that $y_{j}<x_{j}$ (which necessarily exists). Moreover, $y_{j}<\hat{x}_{j}$ since $x_{j} \leq \hat{x}_{j}$. We define $y_{i_{1}}^{\varepsilon}=y_{i_{1}}-\varepsilon, y_{j}^{\varepsilon}=y_{j}+\rho^{j-i_{1}} \varepsilon, y_{i}^{\varepsilon}=y_{i}$ for $i \neq i_{1}, j$. Since $f_{j}^{\prime}\left(y_{j}\right)>f_{j}^{\prime}\left(x_{j}\right)$ and $f_{i_{1}}^{\prime}\left(x_{i_{1}}\right)>f_{i_{1}}^{\prime}\left(y_{i_{1}}\right)$, choosing $\varepsilon>0$ that is small enough (in particular such that $y_{j}+\rho^{j-i_{1}} \epsilon<\hat{x}_{j}$ ) ensures that $\sum_{i \in S} \gamma^{i} f_{i}\left(y_{i}^{\varepsilon}\right)>\sum_{i \in S} \gamma^{i} f_{i}\left(y_{i}\right)$ while $y_{S}^{\varepsilon}$ meets the same constraints as $y_{S}$, which is a contradiction. Since $y_{i_{1}}-x_{i_{1}}<0$, it now follows that $y_{i_{l}}-x_{i_{l}}<0$ successively for $l=2, \ldots, L$.

Moving on to the second step, let $S \subset T \subset N$ and $T<i$. We define $x_{i}^{\prime}=y_{i}^{T \cup i}$ and $x_{j}^{\prime}=y_{j}^{T \cup i}+y_{j}^{S}-y_{j}^{T}$ for $j \in S$. From our first step, $y_{j}^{T \cup i} \leq y_{j}^{T} \leq y_{j}^{S}$ for all $j \in S$. Therefore $0 \leq y_{j}^{T \cup i} \leq x_{j}^{\prime} \leq y_{j}^{S}$ for all $j \in S$ and the consumption plan $x^{\prime}$ for $S \cup i$ satisfies the same constraints as $y_{S \cup i}^{S \cup i}$. Hence, $w(S \cup i) \geq \sum_{j \in S \cup i} \gamma^{j} f_{j}\left(x_{j}^{\prime}\right)$ and

$$
w(S \cup i)-w(S) \geq \gamma^{i} f_{i}\left(x_{i}^{\prime}\right)+\sum_{j \in S} \gamma^{j}\left[f_{j}\left(x_{j}^{\prime}\right)-f_{j}\left(y_{j}^{S}\right)\right]
$$

On the other hand, since $y_{j}^{T \cup i} \leq y_{j}^{T}$ for all $j \in T \backslash S$,

$$
w(T \cup i)-w(T) \leq \gamma^{i} f_{i}\left(x_{i}^{\prime}\right)+\sum_{j \in S} \gamma^{j}\left[f_{j}\left(y_{j}^{T \cup i}\right)-f_{j}\left(y_{j}^{T}\right)\right]
$$

Since $x_{j}^{\prime}-y_{j}^{S}=y_{j}^{T \cup i}-y_{j}^{T}$ and $y_{j}^{T \cup i} \leq x_{j}^{\prime}$ for all $j \in S$, it follows from (22), (23), and the concavity on the rising portion of $f_{j}$, that $w(T \cup i)-w(T) \leq w(S \cup i)-w(S)$. This completes the proof of the lemma. 